Gisele Cova dos Santos Rodrigues

Estudo Comparado da Morfologia da Região do Ozóporo de Opiliões Laniatores

(Arachnida, Opiliones)

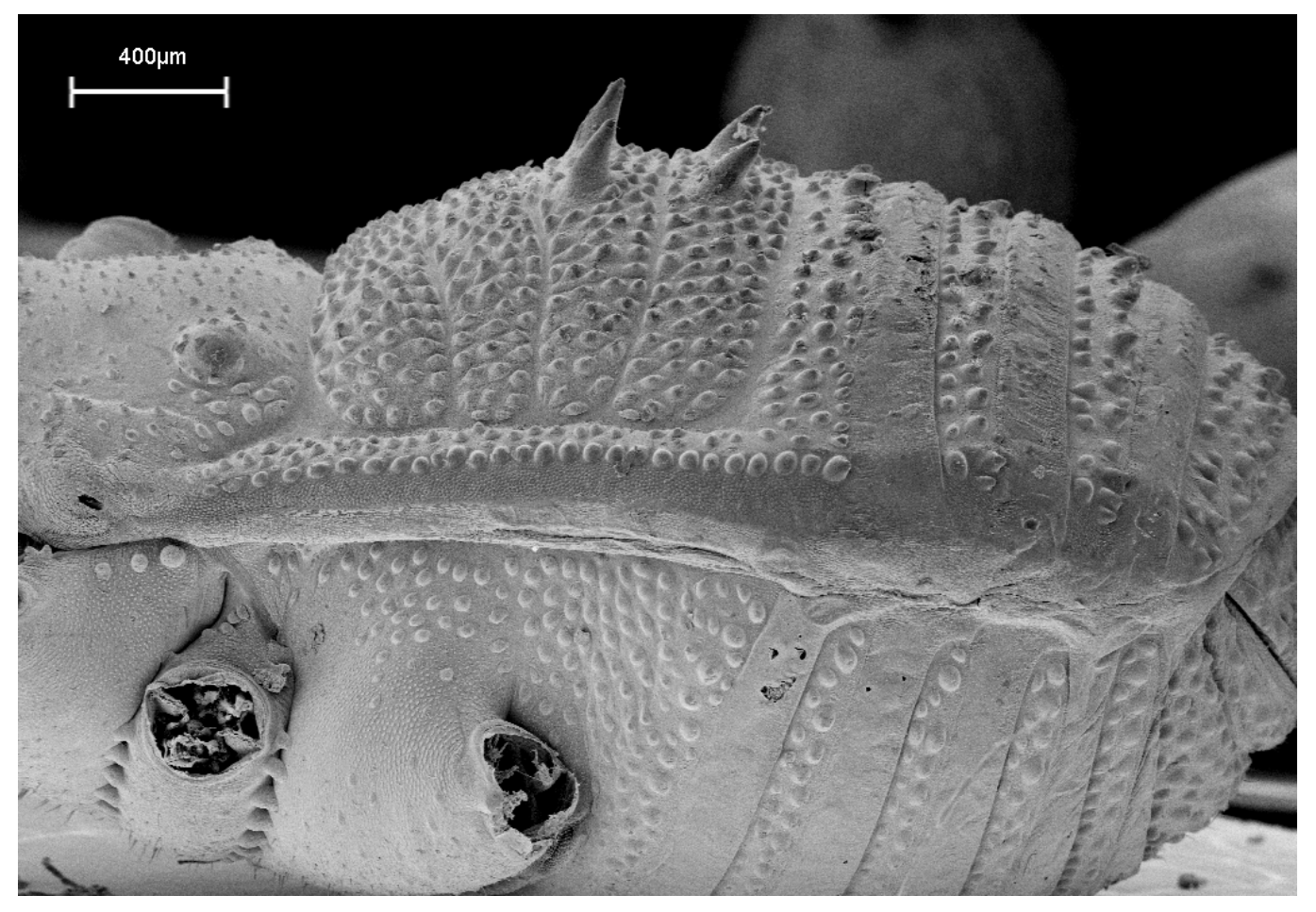

São Paulo

2007 
Gisele Cova dos Santos Rodrigues

\section{Estudo Comparado da Morfologia da Região do Ozóporo de Opiliões Laniatores \\ (Arachnida, Opiliones)}

Dissertação apresentada ao Departamento de Zoologia do Instituto de Biociências da Universidade de São Paulo, para a obtenção de Título de Mestre em Ciências Biológicas (Zoologia).

Orientador: Prof. Dr. Pedro Gnaspini Netto

São Paulo

2007 
Rodrigues, Gisele Cova dos Santos

Estudo Comparado da Morfologia da

Região do Ozóporo de Opiliões Laniatores (Arachnida, Opiliones).

$106 \mathrm{pp}$.

Dissertação (Mestrado) - Instituto de Biociências da Universidade de São Paulo. Departamento de Zoologia.

1. Palavra chave principal: Ozóporo; 2. Palavras-chave secundárias: Morfologia e Laniatores.

I. Universidade de São Paulo. Instituto de Biociências. Departamento de Zoologia.

Comissão Julgadora: 
Como são belas indizivelmente belas essas estátuas mutiladas... Porque nós mesmos lhes esculpimos - com a matéria invisível do ar o gesto de um braço... uma cabeça anelada... um seio... tudo o que lhes falta! 


\section{Agradecimentos...}

Jamais caberia aqui toda minha gratidão para com pessoas, idéias, sentimentos, olhares, ações, muitas vezes abnegações que proporcionaram o desenvolvimento e conclusão deste trabalho. Expresso, pois, parcela de tudo o que sinto para com:

- meu orientador, prof. Dr. Pedro Gnaspini-Netto, pelas idéias, cobranças, críticas, elogios, incentivos, leituras, acompanhamentos em sessões extra de microscopia, paciência com meu desenvolvimento, paciência com meu dia-a-dia, e pelos estímulos para meu progresso;

- ao curador da coleção do MNRJ, prof. Dr. Adriano B. Kury, pela hospitalidade em seu laboratório e gentilezas todas, assim como pelo empréstimo de material;

- ao curador da coleção do MZUSP, prof. Dr. Ricardo Pinto-da-Rocha pelas cobranças, acompanhamentos quando possíveis, críticas e, claro, pelo empréstimo do material;

- Pelo apoio do passado e do presente, Renata e Rodrigo. Pelo abrigo e convivência, Fritz, Bruno, Cris e Musgo. Por tudo sempre, Soninha. Por todo companheirismo e incentivo infinito, às meninas Marie e Marcella! Por proporcionarem campo ao meu trabalho de laboratório. À Marie, que sempre esperou minhas certezas. E sempre esteve pronta!. A Marcella por ajudar em todos os passos, dividindo seus postres, pelas cobranças e pelas análises de tudo um pouco! Por toda leitura!

- aos queridos companheiros no desenvolvimento do meu trabalho: Teté, Sabrina, MBS exLAL-Francês. Ao Marcos Hara, Pudim, que com toda paciência oriental que lhe é cabida tentou me ensinar a como operar um Leiobunum, retirando-Ihe apenas o trocanter, deixando a coxa, as quelíceras, os pedipalpos. Por estar sempre disposto a colaborar, em toda e qualquer situação;

- Com muito carinho, agradeço aos técnicos Enio e Eduardo Mattos que tanto se desdobraram em inúmeros momentos para um trabalho competente e, por isso e pela boa convivência, muito agradável. Assim também, com muito carinho, agradeço a Lara do MZUSP por ter sido tão solícita nas minhas últimas sessões de fotos, também fazendo de tudo o que era possível, por sofrer junto comigo ao ver os bichos maltratados pela limpeza. Ao S. Domingos e ao pessoal da secretaria de pós-graduação que me acolheu com muito respeito, em todo momento, principalmente nos dias de reta final;

- Aos colegas do colégio, por entenderem da importância desse trabalho. Ao Waguinho e Fefoso por sempre terem entendido! Ao carinho de todos meus alunos que já sabem tudo de opiliões, e por me ensinarem tanto. 
- Ao pessoal da faculdade: Alê, Sadao, Leo, Fabitchos, Nel e Nicole! Ao abraço do Martins, ao coração do $\mathrm{Du}$, ao carinho da Pati e do Vinícius, pela distância respeitada, pelas verdades, por todo apoio. Por terem colaborado tanto com tantas visões. À dedicação do Jun pelas ótimas pinças cirúrgicas!! Por toda convivência do Perry, Ednei, Jair, João, Raquel, Natália, Marcinho, Celícia (se precisasse trazia um livro e não ia pra África!), Patona, Boni, Flavinha, Lu, Paulinha, Beto, Nagy, Lígia!! Ao Rafa pela segurança de sempre! Ao Trufa pelo cuidado. Ao pessoal do Arvi por tanto crescimento.

- Agradecimento especial aos amigos de tantas conquistas, de tantas jornadas! Dungão!, Betão, Adilson, Gua, Gabão pelo carinho. Ao Fê, por tudo, por sempre. Sempre um mestre. - Ao PêGodói por ser um porto seguro. Pela sua dignidade, por fazer das minhas conquistas e tropeços, os seus também. Por ter dividido toda alegria e preocupação. Pelo cartão da Edusp com vertebrados dizendo para eu me lembrar das paixões que me motivam. Obrigada por me lembrar quando eu me esqueço. Pelas broncas, pela paciência. Claro, por toda parte prática também!! Escalas, apoio na bibliografia e pdfs...

- A todos da família: minha vó e sua visão de mundo, meu pai, por respeitarem minhas correrias, Buzuzu por estar sempre pronta, e, em especial, agradeço a minha mãe, Dona Pê, por toda revisão do trabalho, por toda conquista e sofrimento compartilhados

- A tantos e por muito. Enfim, a todos que se interessaram tentando me ajudar a trabalhar com o “obulhão?", “opulhão?”, “olipião, né?”... 
Índice

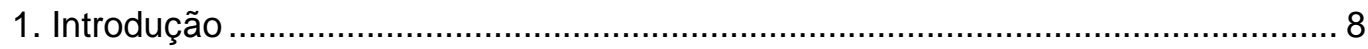

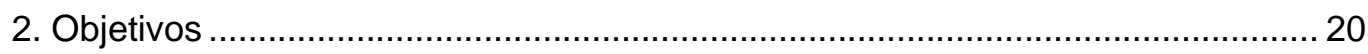

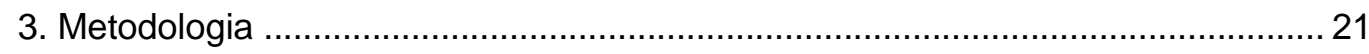

4. Resultados e Discussão ........................................ Erro! Indicador não definido.

4.I. Descrição das diversas morfologias....................... Erro! Indicador não definido.

4.I.1. Definição dos caracteres analisados .................. Erro! Indicador não definido.

4.I.2. Descrição da morfologia da área do ozóporo ..... Erro! Indicador não definido.

4.II. Discussão ........................................................ Erro! Indicador não definido.

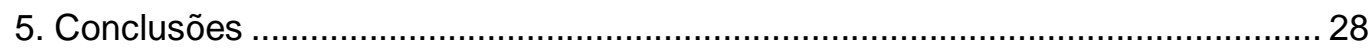

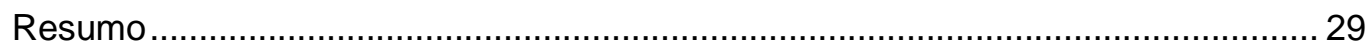

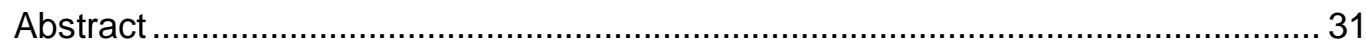

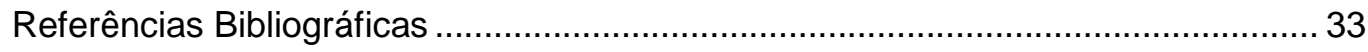




\section{Introdução}

Ao longo dos anos de estudo da vida animal, percebe-se que há o desenrolar de uma corrida armamentista evolutiva (Krebs \& Davies, 1966; Edmunds, 1974) entre as adaptações apresentadas por uma presa qualquer em face às adaptações do predador e, inclusive, sendo válido o contrário: a contra-adaptação do predador eventualmente selecionada pela morfologia, fisiologia ou comportamento da presa. Populações de predadores apresentam continuadamente métodos eficientes para localização e captura das presas, enquanto estas últimas podem apresentar métodos cada vez mais eficientes em tornarem-se imperceptíveis ou mesmo indesejáveis, seja por resposta mecânica ou química, de origem endógena ou exógena.

Dadas certas condições ambientais, físico-químicas de certo habitat, e biológicas, os animais podem apresentar certos padrões de defesa, o que permite o incremento na taxa de sobrevida do indivíduo, ou mesmo do grupo ao qual ele pertence.

Edmunds (1974) categoriza o estudo da defesa nos animais sob a perspectiva da relação presa/predador, se é o caso de uma relação em potencial ou se é de fato real.

As adaptações das presas que visam a diminuir a possibilidade do encontro são consideradas mecanismos de defesa primária. Tais adaptações ocorrem no sentido de diminuir a probabilidade do predador em localizar as presas ou identificá-las como tal, ou mesmo do predador, apesar de reconhecer o indivíduo como presa, evitar o combate por relacioná-lo a uma experiência anterior negativa.

Todavia, visando a incrementar as chances de sobrevivência individual em face de um possível encontro com um predador, muitos animais apresentam artifícios para evitar a ocorrência da predação em si. Esses 
artifícios foram considerados como mecanismos de defesa secundária por Edmunds (1974). Podem ocorrer apenas pela percepção da aproximação de um indivíduo que é potencialmente um predador, significando apenas a acentuação de uma defesa primária, por exemplo.

Encontram-se na natureza e também na literatura vários casos de mecanismos de defesa primária e secundária. De modo geral, Edmunds (1974) destaca as seguintes categorias de defesa primária: o fato de o animal apresentar coloração críptica, anacorese, aposematismo ou mimetismo. Como mecanismos de defesa secundária, ou seja, apresentados após a identificação do predador, destacam-se a tanatose, que é o comportamento de fingir-se de morto, diminuindo o poder de atração para si, aumentando as chances do predador de se desinteressar; o comportamento deimático, ou a adoção de posturas, comportamentos ou de sons que intimidem o predador; a oferta de áreas do corpo que não são vitais, o que pode permitir a subseqüente fuga da presa; apendotomia, fuga acelerada ou mesmo qualquer tipo de retaliação que for possível em um contato direto: retaliação mecânica através de pinças, espinhos, armaduras, quelíceras, pedipalpos, ou mesmo uma retaliação baseada em substâncias químicas.

A partir de revisão da literatura, observa-se substancial volume de registros dentre os indivíduos da Ordem Opiliones, de comportamentos categorizados, a priori, como de defesa. Todas as classificações supracitadas de Edmunds (1974) já foram observadas dentre os opiliões. Hara \& Gnaspini (2007) sintetizaram muitos dos vários estudos de tais métodos de defesa no grupo em questão. Entretanto, cabem ainda algumas poucas adições a tal resumo: os comportamentos de se retrair em algum local protegido frente a potencial presença de um predador, jogar-se no chão permanecendo imóvel, objetivando passar despercebido também foram 
observados em Stygnomma spinifera, por Duffield et al. (1981). Gonisosoma catarina vem corroborar a afirmação de que os machos adultos da subfamília Goniosomatinae aplicam o movimento de pinça, o "beliscão", utilizando-se das armaduras do fêmur IV, visto que também se utilizam de tal mecanismo (Machado et al., 2002).

Ao que tudo indica, as cerca de 6000 espécies descritas até o momento (Machado et al., 2007), apresentam capacidade de síntese de compostos químicos. Isso porque, segundo Shultz (1990), apresentar um par de glândulas exócrinas é um caráter sinapomórfico para a ordem. A posição da abertura deste órgão no prossoma, entretanto, é variável de acordo com a subordem analisada.

A ordem Opiliones é subdividida em quatro subordens, de acordo com o estudo filogenético proposto por Giribet et al. (2002): Cyphophthalmi, Eupnoi, Dyspnoi e Laniatores. A primeira subordem, com um pouco mais de 100 espécies distribuídas em seis famílias, possui representantes em todos os continentes e grandes ilhas, à exceção da Antártica (Giribet \& Kury, 2007). Seus exemplares são ricamente esclerotizados, e de medidas similares às de ácaros. A abertura da glândula, o ozóporo, localiza-se sobre o ozóforo, um tubérculo localizado no dorso, próximo à margem do prossoma. Segundo Juberthie (1961a,b), são os representantes desta subordem os que possuem um sistema muscular mais elaborado associado à glândula e seu respectivo ducto: há um feixe de músculos ventrais que abre o ducto, outro que é o grupo muscular do próprio ducto e um terceiro feixe que comprime a glândula.

A subordem Eupnoi inclui as superfamílias Phalangioidea, com cinco famílias, e Caddoidea, com apenas uma família (Shear, 1982). Ao contrário dos representantes de Cyphophthalmi, os opiliões Eupnóicos possuem corpo 
delgado e longas pernas. São muito comuns a ambos os Hemisférios e hoje contam com 1780 espécies descritas (Machado et al. 2007).

A terceira subordem reconhecida por Giribet et al. (2002) é a dos opiliões relativamente mais robustos, restritos ao Hemisfério Norte, encerrando as superfamílias Ischyropsalidoidea e Troguloidea, com três e quatro famílias, respectivamente. Esta subordem apresenta 290 espécies descritas. (Machado et al., 2007).

Um caráter marcante que permite uma identificação mais grosseira entre essas duas subordens previamente descritas é o dente da quelícera: diafanizado ou não. Se apresentar o dente diafanizado, é um representante de Dyspnoi, caso contrário, trata-se de um Eupnoi. (Pinto-da-Rocha \& Giribet, 2007).

Contudo, indivíduos das duas subordens são muito similares ao que se conhece da morfologia da glândula secretora e de sua abertura. $O$ sistema muscular constituinte da estrutura glandular, tanto o feixe ventral, responsável em abrir o assoalho do ozóporo, quanto o feixe que pressiona a própria glândula em Cyphophthalmi, estão ausentes, o que pressupõe um outro mecanismo de expulsão da secreção química, seja ele nervoso ou envolvendo outros grupos musculares (Juberthie, 1961 a,b; Holmberg, 1970; Clawson, 1988). Já o ozóporo, tanto de Eupnoi quanto de Dyspnoi, localizase na margem antero-lateral, próximo à coxa I (Juberthie, 1961 a).

A quarta e última subordem, Laniatores, é objeto de estudo do presente trabalho, e é a que conta com maior número de espécies descritas até o momento, são 3748. Distribuem-se pelas áreas tropicais e temperadas, principalmente do Hemisfério Sul. A subordem dos Laniatores é ainda subdividida em duas infra-ordens: Insidiatores e Grassatores. Os representantes dos Insidiatores possuem uma garra bífida nas pernas III e 
IV, e ainda um sistema muscular entre as placas ventrais e dorsais do pênis, enquanto os dos Grassatores possuem duas garras de inserções independentes nos tarsos III e IV, sem qualquer musculatura peniana. Travunioidea e Triaenonychoidea representam os Insidiatores, enquanto Zalmoxoidea, Samooidea, Phalangodoidea, Epedanoidea e Gonyleptoidea são as superfamílias de Grassatores, que contém, por enquanto, 26 famílias estabelecidas (Giribet \& Kury, 2007).

Como todos os opiliões possuem um par de glândulas no prossoma, é esperado que todos produzam a secreção química. Originalmente chamada de gonyleptidina por Estable et al. (1955), é amplamente utilizada como uma ferramenta para suas defesas, e seu potencial já foi testado várias vezes, inclusive lançando mão de diversos possíveis predadores, tais como várias espécies de formigas e algumas aranhas, por exemplo, tanto testando com o indivíduo secretando a substância de defesa, quanto à irritabilidade da substância por si só (Cloudsey-Thompsonm 1968; Blum \& Edgar, 1971; Eisner et al., 1971; Eisner et al., 1978; Jones et al., 1977; Blum, 1981; Acosta et al., 1993; Eisner et al., 2004; Machado et al., 2000, 2002, 2005).

A biossíntese de compostos químicos é amplamente utilizada em Arthropoda como método de defesa primária, quando traz ao predador a lembrança de uma experiência desagradável dada uma prévia intoxicação ou impalatabilidade ocorrida pelo consumo de um animal que apresentava defesas químicas, ou ainda dada a possível herança que o distancia de uma presa com certas características aposemáticas. Também é utilizada como defesa secundária quando da secreção de substâncias tóxicas a outro ser vivo no momento da predação em si.

Desde a década de 1950, foram realizados vários estudos abordando a caracterização química dos compostos produzidos pelos diversos opiliões, 
com ênfase às ordens de Laniatores e Eupnoi. Através de técnicas de cromatografia gasosa, espectroscopia, espectrometria de massa e outras técnicas que sofreram diversas melhorias ao longo das décadas, foi possível a identificação precisa de vários compostos.

Laniatores, de forma geral, produzem quinonas metiladas (Estable et al., 1955; Fieser \& Ardao, 1956; Eisner et al., 1971, 2004; Acosta et al., 1993; Gnaspini \& Cavalheiro, 1998) e, eventualmente alguns tipos de fenóis (Eisner et al., 1977, 1978; Roach et al., 1980; Duffield et al., 1981). Ekpa et al. (1984) registraram em Sclerobunus robustus, espécie da família Triaenonychidae, a ocorrência de substâncias que divergem do que se sabe que muitos outros opiliões produzem, tais como bornil-propionato, bornil-acetato, feniltilamina, nicotina e traços de limoneno e canfeno. Hara et al. (2005) realizaram extensa análise da composição química das secreções de defesa de 22 espécies pertencentes à família Gonyleptidae, identificando 19 novos compostos químicos para o grupo.

A biossíntese de quinonas como forma de defesa é comum entre os invertebrados, especialmente em insetos e milípedes (Aneshansley \& Eisner, 1969; Eisner \& Meinwald, 1966, Eisner, 1970; Weatherston \& Percy, 1970), mas 2-etil-1,4-benzoquinona nunca havia sido registrada em Opiliões; 2,3,5trimetil-1,4-benzoquinona, por exemplo, nunca havia sido registrada em outro grupo (Eisner et al., 1977). Há inclusive algumas substâncias como 4,6dimetil-6-noneno-3-ona (Jones et al., 1976), 2-metil-5-etilfenol (Eisner et al., 1977) e 2,3,4-trimetilfenol (Roach et al., 1980), dentre outras, que nunca haviam sido encontradas na natureza.

Eupnoi e Dyspnoi, por sua vez, comumente sintetizam cadeias curtas acíclicas com cetonas, aldeídos e álcoois ou também naftoquinonas (Blum et al., 1968; Blum \& Edgar, 1971; Jones et al., 1976, 1977; Meinwald et al., 
1971; Wiemer et al., 1978, Ekpa et al, 1985). Jones et al. (1977) também chamaram a atenção para o fato de haver variabilidade na composição da secreção em indivíduos diferentes, apesar de serem pertencentes à mesma espécie.

Tradicionalmente, o uso da substância secretada nos opiliões é associado à defesa: tanto que a glândula é comumente chamada de glândula de defesa ou repugnatória. Por outro lado, com um pouco mais de cuidado em outros tipos de comportamento, pode-se reconhecer algumas outras situações em que a substância poderia ser útil: eventualmente como feromônios, podendo estimular e/ou inibir comportamentos de cópula, fuga ou ataque, ou mesmo como uma substância de demarcação de trilha ou desova, ou até limpeza de uma área. Bishop (1950) já se questionava quanto à possibilidade das substâncias serem utilizadas para comunicação. Estable \& Fieser (1955), em trabalho pioneiro de identificação da substância, reconhecem suas propriedades antibacterianas, fungicidas e inclusive sua toxicidade frente a protozoários, tal como Trypanosoma cruzi. Blum \& Edgar (1971) reconhecem a mesma substância química em formigas, porém utilizada como feromônio. Clawson (1988), acredita que fêmeas marcam áreas que lhes serão úteis como sítios de desova, possivelmente diminuindo o risco de outra fêmea vir a se apropriar daquele espaço. Rodriguez \& Guerrero (1976), Mora (1990) e Machado et al. (2002) acreditam haver algum tipo de demarcação em áreas de desova ou em possíveis trilhas, dada a alta especificidade de reconhecimento dos adultos ou de retorno aos ninhos, quando previamente retirados. Também vale a pena destacar a não utilização da substância de defesa por pais e/ou mães que estejam em processo de cuidado parental, à exceção de Acutisoma proximum (v. em Ramires \& Giaretta, 1994), nem tampouco em brigas por ninhos ou por 
fêmeas, ou por machos (Rodriguez \& Guerrero, 1976; Mora, 1990; Machado \& Oliveira, 1998; Willemart \& Gnaspini, 2004). Willemart (2002) registrou pela primeira vez a ocorrência da secreção da substância de defesa contra um outro opilião como parte de uma competição por alimento: o evento ocorreu entre Neosadocus varibialis, como um mecanismo adicional à defesa mecânica. A agressão ocorreu contra um outro opilião, também pertencente à família Gonyleptidae, Heteromitobates discolor.

Adicionalmente, há evidências acerca do uso dessas substâncias químicas em e para a formação de agregados. Os indivíduos que participam de um grupo social eventualmente apresentam vantagens evolutivas, uma vez que, entre outras razões, há possibilidade de uma maior concentração de substâncias repelentes aos agressores, ou de menor probabilidade de predação frente a tantos indivíduos (Holmberg, 1986; Machado \& Vasconcelos, 1998; Machado et al., 2000; Machado, 2002). Também a eliminação de tais compostos químicos pode agir como sinal de alerta em agregados, pelo menos de Goniosoma aff. proximum, frente à situação de perigo (Machado et al., 2002). O que também foi registrado em agregados multi-específicos no trabalho de Machado \& Vasconcelos (1998) foi a secreção da substância de defesa por Holoversia nigra, e não por Despirus montanus (Mitobatinae), nem Eugyndes sp. (Pachylinae). A vantagem pode residir no aumento da taxa de sobrevivência, visto que havendo o efeito de diluição em um agregado, as chances de serem predados são diminuídas.

Há algumas formas distintas dos animais secretarem suas substâncias sintetizadas nas glândulas torácicas, quando em situação de perigo. Encontram-se na literatura algumas observações (Bristowe, 1925; Capocasale \& Bruno-Trezza, 1964; Edgar, 1971) e várias descrições desse 
comportamento (Lawrence, 1938; Juberthie, 1961; Eisner et al., 1971, 1977; Duffield et al., 1981, Maury, 1987; Clawson, 1988).

Dentre toda a diversidade da fauna de opiliões, até o final do século passado, havia pouco mais do que duas dezenas de espécies com o comportamento de defesa química relatado. Entretanto, o simples relato do comportamento e/ou da morfologia e composição química não permitiria qualquer predição filogenética, nem qualquer outra conjectura que poderia viabilizar, por exemplo, a descrição de comportamento de todo um grupo.

O primeiro trabalho a sistematizar o estudo da secreção da substância de defesa foi o de Acosta et al. (1993), quando, além de sumarizar as composições químicas das secreções reconhecidas até a data em questão, também reconheceu três padrões de se efetivar a descarga das substâncias químicas. Apesar das compilações, não propôs conseqüentes relações entre as composições químicas das secreções e os padrões de comportamento relatados.

Acosta et al. (1993) classificaram três formas básicas de se eliminar a substância de defesa e a formulação das categorias ocorreu segundo uma crescente otimização na utilização da defesa química. Considerou como primeiro padrão de secreção a simples evaporação a partir da abertura da glândula, ou a formação de uma gota na própria área do ozóporo. Juberthie (1961b) não reconheceu a eliminação de líquido, mas percebeu odor característico da substância de defesa de Rhampsinitus levi. Em tantos outros, notou-se a formação de uma gota na área do ozóporo, sem o posterior escoamento, tal como foi registrado para Phalangium opilio, Querilhacia querilhaci, Peltonychia clavigera (Juberthie, 1961b) e Adaeulum robustum (Lawrence, 1938). 
O segundo padrão sugerido pelos pesquisadores é o que considera algum tipo de mecanismo que permita ampliar a área de evaporação, seja emitindo finos jatos, como fora registrado em Leiobunum formosum e Leiobunum speciosum (Blum \& Edgar, 1971), ou com o escoamento da substância de defesa através de canais ou uma série de tubérculos presentes nas margens do escudo dorsal. Metabiantes leighi, Scotolemon lespesi, Scotolemon doriae, Scotolemon Iucasi, Stynomma spinifera e Pachyloidellus goliath (Lawrence, 1938; Juberthie, 1961b; Duffield et al., 1981 e Acosta et al., 1993) registraram escoamento da secreção de defesa através de canais laterais, ao passo que em Zygopachylus albomarginis (Cokendolpher, 1987) tal escoamento também foi registrado, mas por meio de uma série de tubérculos.

Por fim, Acosta el al. (1993) consideram também o comportamento de direcionar a secreção diretamente contra o agressor como um padrão possivelmente adotado, otimizando, portanto, tanto quanto possível, a toxicidade da substância de defesa. Cosmetidae é uma família da subordem dos Laniatores em que seus representantes demonstraram o comportamento de molhar as regiões apicais dos tarsos e transpor a secreção diretamente ao agressor, assim como os indivíduos estudados de Sironidae, família de Cyphophthalmi (Juberthie, 1961; Eisner et al., 1971, 1977). Uma outra maneira que direciona a substância potencialmente tóxica contra o agressor é a eliminação da secreção sob a forma de um jato, tipicamente encontrado em alguns opiliões da família Triaenonychidae (Lawrence, 1938; Maury, 1987).

Gnaspini \& Cavalheiro (1998) por sua vez, chamaram a atenção para a variação quanto ao local da formação de uma gota contendo a solução química de defesa. Destaca que em Goniosoma spelaeum, assim como em 
Zygopachylus albomarginis, a gota é formada apenas na região próxima à coxa IV, o que em $Z$. albomarginis seria o equivalente aos espinhos localizados nas margens latero-posteriores do escudo dorsal. Os autores sugerem que a morfologia mais complexa da área do ozóporo de $G$. spelaeum possa ter relação com tipo de comportamento, que é o de eliminar a substância de defesa química sob a forma de um jato.

As relações entre os padrões de comportamento de secreção da substância de defesa e a morfologia da região do ozóporo finalmente foram obtidas por Hara \& Gnaspini (2003), que observaram a morfologia e o comportamento de defesa química em 33 espécies pertencentes à família Gonyleptidae. Com base nas hipóteses propostas de relações entre as subfamílias de Gonyleptidae (Pinto-da-Rocha, 2002), esses autores notaram que os grupos basais apresentavam uma abertura mais simples, menos ornamentada, e que o animal apresentava o comportamento de emitir uma gota da substância de defesa, a qual evaporava junto à abertura ou era transferida por canais laterais e se espalhava pelo corpo dos animais, aumentando a área de evaporação (Lawrence, 1938; Duffield et al., 1981; Cokendolpher, 1987). Perceberam também que havia uma progressão na complexidade morfológica e funcional da área do ozóporo, ou nas estruturas acessórias, ao comportamento de eliminação da substância de defesa, até atingir o padrão considerado mais derivado, no qual a abertura apresenta-se na forma de um sulco alongado em cuja porção mediana ocorre uma ampliação da abertura pela qual pode haver a eliminação de um jato direcionado ao agressor (Lawrence, 1938; Gnaspini \& Cavalheiro, 1998).

A partir do trabalho de Hara \& Gnaspini (2003) percebe-se que a morfologia da área de abertura da glândula é, muitas vezes, determinante sobre o comportamento adotado para eliminação da substância de defesa. $\bigcirc$ 
presente estudo teve como objetivo o levantamento de dados morfológicos da área do ozóporo de diversas famílias da ordem Laniatores. Conhecer as variações e os padrões de estruturas constituintes da região em questão entre tantos grupos permite o acréscimo de informações à literatura acerca de um grupo com tamanha diversidade, e restrito histórico de pesquisa.

Ainda, uma vez que é esperado que o comportamento adotado para a secreção das substâncias químicas seja também condicionado à morfologia da área do ozóporo (dentre outras razões, pois o comportamento pode variar dependendo, por exemplo, da insistência do predador, da disponibilidade de água, do fato de fazer ou não parte de um agregado), conhecer tal morfologia significa a possibilidade de conhecer os tipos de comportamentos possíveis para secreção química. E identificar essas possibilidades é uma via que possibilita compreender, de forma mais ampla, o quando e os porquês do uso das substâncias químicas. 


\section{Objetivos}

O presente estudo teve por objetivos conhecer mais detalhadamente as estruturas e a morfologia da área do ozóporo de diversas famílias de Laniatores, buscando com isso adicionar informações à literatura acerca de famílias que não Gonyleptidae, famílias relativamente pouco conhecidas.

Também é parte dos objetivos desse trabalho levantar informações sobre a morfologia para que, posteriormente se tenha argumentos e se possa inferir qual tipo de comportamento de defesa o indivíduo de uma certa linhagem pode desenvolver. 


\section{Metodologia}

Visando englobar tantas famílias de Opiliões Laniatores quanto possíveis, o presente estudo contou com empréstimos de material biológico das coleções de aracnologia do Museu de Zoologia da Universidade de São Paulo (MZUSP), bem como do Museu Nacional do Rio de Janeiro (MNRJ). E, em colaboração de Abel Peréz González, pesquisador do MNRJ, quando possível, a presença de fêmea e macho para comparação da morfologia da região do ozóporo, sob o prisma da intraespecificidade. Além de permitir a observação de possível dimorfismo sexual nesta região, talvez como conseqüência de padrões diferenciados de defesa comportamental condicionado pelo sexo, uma vez que os machos de algumas espécies apresentam estruturas morfológicas de defesa, tais como pernas IV mais armadas.

As 16 famílias de Laniatores abordadas estão relacionadas na tabela 1, assim como possíveis informações adicionais sobre a taxonomia de cada espécie.

Houve preparação da grande maioria dos animais, todavia os exemplares de Biantidae, Crosbyella, gênero de Phalangodidae, Podoctidae, Stygnommatidae e Biacumontia, gênero de Triaenonychidae, foram emprestados a partir da coleção pessoal de A.B. Kury, já fixados em stubs previamente preparados na década de 1980 pelo próprio. A técnica utilizada na época é diferente da adotada na presente preparação, e os animais foram mantidos fora de estufa, em caixas fechadas. 
Tab. 1: Relação das famílias estudadas no presente estudo apresentando, quando identificado, a subfamília, o gênero e, talvez, a espécie do animal abordado. Ainda, qual a origem do material em questão. O material biológico foi emprestado de duas coleções aracnológicas: MZUSP e MNRJ. * Ainda, contou com material previamente preparado por A. B. Kury (AMNH 1994). ${ }^{* *}$ LEA: Material de coleção pessoal de P. Gnaspini.

\begin{tabular}{|c|c|c|c|c|}
\hline Família & Sub-Familia & Gênero & Espécie & Origem \\
\hline Agoristenidae & Leiosteninae & Trinella & Trinella venezuelica & MZUSP: 14.574 \\
\hline Agoristenidae & Agoristeninae & & Spp.1 & MNRJ \\
\hline Biantidae & Biantinae & Metabiantes & Metabiantes sp & Stub AMNH 1994 \\
\hline Biantidae & Stenostygninae & Caribbiantes & Caribbiantes sp & Stub AMNH 1994 \\
\hline Cosmetidae & Comestinae & Cosmetus & Cosmetus variolosus & MZUSP: 280 \\
\hline Cosmetidae & Comestinae & Cynortellula & Cynortellula bimaculata & MNRJ: 4.619 \\
\hline Cosmetidae & Comestinae & Cynorta & Cynorta conspersa & MNRJ: 4.560 \\
\hline Cranaidae & Cranainae & Santinezia & Santinezia sp. & MZUSP: 18.862 \\
\hline Cranaidae & Cranainae & Santinezia & Santinezia serratotibialis & MNRJ: 16.120 \\
\hline Epedanidae & Arcobuninae & Pseudobiantes & Pseudobiantes sp. & MNRJ \\
\hline Escadabiidae & & Baculigerus & Baculigerus sp. & LEA \\
\hline Escadabiidae & & Baculigerus & Baculigerus milenal & MNRJ \\
\hline Fissiphalidae & & Fissiphallius & Fissiphallius sp. & MNRJ \\
\hline Manaosbiidae & & Spp.1 & Spp.1 & MZUSP: 21.461 \\
\hline Manaosbiidae & & Spp. 2 & Spp. 2 & MZUSP: 15.917 \\
\hline Minuidae & & Metakimula & Metakimula sp. & MNRJ \\
\hline Phalangodidae & Phalangodinae & Crosbyella & Crosbyella spinturnix & Stub AMNH 1994 \\
\hline Phalangodidae & & Scotolemon & Scotolemon sp. & MNRJ \\
\hline Podoctidae & & Melanoctis gen novo & Melanoctis sp. & Stub AMNH 1994 \\
\hline Samoidae & & Pellobunus & Pellobunus sp. & MNRJ \\
\hline Samoidae & & Neoscotolemon & Neoscotolemon sp. & MNRJ \\
\hline Stygnidae & Heterostygninae & Stygnoplus & Stygnoplus clavitibialis & MZUSP: 18.867 \\
\hline Stygnidae & Stygninae & Stygnus & Stygnus multispinosus & MZUSP:15.166 \\
\hline Stygnidae & Stygninae & Protimesius & Protimesius longipalpis & MZUSP: 14.216 \\
\hline Stygnidae & Stygninae & Protimesius & Protimesius longipalpis & MZUSP: 14.276 \\
\hline Stygnommatidae & & Stygnomma & Stygnomma bonaldoi & Stub AMNH 1994 \\
\hline Triaenonychidae & Adaeinae & Larifugela & Larifugela afra & LEA \\
\hline Triaenonychidae & Adaeinae & Larifugela & Larifugella afra & MNRJ: 11.414 \\
\hline Triaenonychidae & Sclerobuninae & Sclerobunus & Sclerobunus robustus Packard & MZUSP: 20.640 \\
\hline Triaenonychidae & Triaenonychinae & Biacumontia & Biacumontia sp. & Stub AMNH 1994 \\
\hline Zalmoxidae & & Soledadiella & Soledadiella macrochelae & MNRJ \\
\hline Zalmoxidae & & Cippanus & Cippanus adornus & MNRJ: AK 210 USNM \\
\hline
\end{tabular}


Com o auxílio de pinças utilizadas em microcirurgias odontológicas, e material de dissecção, as pernas foram extirpadas com uma incisão na ligação entre o trocanter e a coxa, na região da articulação transversal. A princípio, as pernas foram seccionadas na região central dos fêmures, sendo que, em alguns exemplares preparados no início do trabalho, nem o trocanter foi retirado. Com a análise dos resultados, percebeu-se que muitas vezes tal trocanter impedia a vista lateral ou dorsal da região do ozóporo. Esta metodologia sofreu melhorias com as subseqüentes preparações, inclusive com a retirada dos trocanteres I, II e III. Houve casos de se retirar a apófise presente na coxa II ao se perceber, ainda sob lupa, que era uma potencial cobertura da área do ozóporo, o que impediria sua visualização. A área anterior dos animais foi preservada para se manter o máximo de estruturas passíveis de análises morfológicas futuras e, além disso, para se manter a fisionomia natural do animal, tanto quanto possível. Muitas vezes as pernas IV foram mantidas até a área mediana do fêmur, de modo a se permitir a melhor manipulação preservando-se a integridade do corpo do animal, bem como a colocação do indivíduo no suporte metálico ("stub"), procurando-se manter as áreas dorsal e ventral visíveis para a sessão de fotos nos microscópios eletrônicos de varredura. Os indivíduos eram dispostos com o opérculo anal preso a um dos lados de uma fita dupla face, sendo a outra face aderida diretamente ao stub.

Após a sessão cirúrgica, seguia-se o processo de limpeza do indivíduo. De forma geral, seguiu-se à metodologia utilizada em Pinto-daRocha (2002), mas com o passar do tempo e análise das fotos, algumas modificações foram adotadas. A idéia geral era a de limpeza do exoesqueleto de quaisquer impurezas, inclusive ectoparasitas, por um sistema de lavagem em banho de ultra-som, utilizando-se, para isso, uma solução de detergente 
comercial a 33\%, renovado a cada troca. Foram em média três sessões de quinze minutos cada, havendo renovação da solução de detergente a cada uma. Tal processo permite o descolamento de placas de gordura e torna as impurezas adsorvidas instáveis, facilitando o processo de retirada com jatos leves dessa solução com micropissetas. Entretanto, dependendo da resistência do animal, três sessões de quinze minutos em ultra-som é pouco para a retirada de impurezas, ou também podem vir a se tornar extremamente agressivas. Infelizmente, dada a escassez de material biológico disponível, não foi possível aplicar metodologias de limpeza diferentes, testando qual seqüência é mais adequada para cada animal. Preconizou-se, portanto, a utilização do método padrão com sensíveis alterações de tempo no ultra-som balizadas por percepção tátil ao se manipular o animal sob lupa. Ou seja, infelizmente há fotos de animais muito interessantes que retratam um indivíduo castigado pela metodologia que não pôde ser testada em uma amostra representativa.

Finalizado o processo de limpeza realizado no ultra-som, deixava-se o material repousando nesta solução por cerca de 12h, exatamente visando à retirada de demais impurezas que porventura permaneciam insistentemente adsorvidas em estruturas como cerdas e sensilas.

Após todo o processo de cirurgia e limpeza, seguiu-se a série de desidratação sucessiva. Como os animais passaram por tanta solução de detergente comercial, fez-se necessária a remoção desses resíduos: com uma série de imersão em água destilada. Tal série consiste de três trocas de 10 minutos cada.

A desidratação sucessiva que é advinda com a remoção dos resíduos de detergente, consiste em submersão em soluções de álcool de crescentes concentrações. A primeira delas é de álcool a 70\%, concentração esta de 
conservação do espécime. Em seguida, o animal é transferido para uma solução a $80 \%$, então para outra a $90 \%$ e, por fim, o animal é imerso em álcool absoluto. A série de desidratação envolve trocas de solução a cada 15 minutos, à exceção do álcool absoluto em que se propõem três trocas, cada uma permitindo 10 minutos de submersão.

Ao término da desidratação, o animal permanece em álcool absoluto por cerca de $12 \mathrm{~h}$. É então conduzido para um processo de aquecimento até atingir um ponto crítico quando o álcool vaporiza, em "Critical Point Dryer BAL-TEC CPD 30". Após o ponto crítico foram metalizados todos os exemplares reportados nessa dissertação, o que significa que todos receberam uma carga de ouro em pó, impedindo, desta forma, a suscetibilidade da carapaça aos feixes de elétrons. O equipamento utilizado para tal metalização foi o "Sputter Coater Balzer SCD 50". Há que se complementar ainda que, certa feita, houve a tentativa de se preservar um exemplar e analisá-lo sem tal banho de ouro. Infelizmente, este indivíduo, um animal italiano, Ischyropsalis, teve seu exoesqueleto perfurado pelo feixe de elétrons em alguns pontos.

Ao banho de ouro segue-se a etapa de montagem dos stubs: com uma fita dupla-face ou mesmo com cola de prata, fixam-se os animais na superfície do stub, que foi identificado com números distintos e individualizados na sua superfície inferior, ou mesmo através de uma marcação realizada com pontas de agulhas em esmalte fresco. Algumas vezes, a manipulação foi prejudicada pela ausência de estruturas que não seriam analisadas como suporte, tal como os fêmures IV.

Finalmente, as sessões de fotos foram realizadas em dois ambientes distintos, a saber: Laboratório de Microscopia Eletrônica do Instituto de Biociências da Universidade de São Paulo, no aparelho Digital Scanning 
Microscope (DSM) 940, Zeiss, e com o aparelho LEO-440 Zeiss do Museu de Zoologia da Universidade de São Paulo. Todas as sessões foram monitoradas pelos técnicos responsáveis pelos equipamentos. Nas sessões de fotos, buscou-se o cuidado com a manutenção do padrão da foto, procura pela região do ozóporo em ambos os lados do animal, para o caso de visualizar com maior clareza a ornamentação ou mesmo estrutura. Por isso os animais eram dispostos de forma a ficar com o opérculo anal colado ao stub. Nas fotos, também foi padronizada a seqüência de posições: foto dorsal; foto ventral se possível, tentando evidenciar-se os canais ventrais; foto lateral com e sem grande aumento, permitindo vista geral dos canais laterais, e detalhamento da carapaça na região do ozóporo.

As imagens digitais obtidas a partir do laboratório de microscopia eletrônica do IB-USP foram gravadas em zip disk e, por segurança, consolidadas, a posteriori em mídias ópticas do tipo CDs graváveis (cd-r). Já as imagens obtidas a partir do MZUSP foram armazenadas diretamente em cd-r. Todas as fotos foram tratadas visando à obtenção de otimização no contraste e brilho, através do software de manipulação de imagens, Adobe Photoshop CS.

Os animais já observados foram armazenados em estufas do IBUSP a $40^{\circ} \mathrm{C}$

A terminologia que consta nesta dissertação foi fundamentada em Lawrence (1938), Murphree (1988), Acosta et al. (1993), Gnaspini \& Cavalheiro (1998) e Hara \& Gnaspini (2003).

Vale ressaltar que houve duas tentativas de outros métodos, ambas não utilizadas para o levantamento de dados para este trabalho: a utilização de microscópio eletrônico confocal no Instituto Butantan, e o da análise histológica do corte da região do ozóporo. O uso de confocal não pareceu 
trazer novidades ou incrementos ao uso da microscopia eletrônica de varredura convencional. Ainda que traga uma visão tridimensional da estrutura, não foi possível manipular o material de forma a se obter melhor ângulo da abertura do canal da glândula de defesa. A histologia por sua vez, parece ser uma metodologia interessante a um trabalho mais detalhado enfocando a morfologia, especificamente da área do ozóporo. Mas percebeuse que, para atingir sucesso nessa metodologia, seriam necessários alguns testes em alguns indivíduos, o que inviabilizou sua prática neste trabalho, dada a utilização de, muitas vezes, material pouco comum nas coleções de aracnologia por ora abordadas. 


\section{Conclusões}

A morfologia da região do ozóporo apresenta alguns padrões, tais como a posição de abertura, o direcionamento do ozóporo, seu formato, os tipos de apófise que surgem, os tipos de tegumento, entre outros, os quais podem ser utilizados para caracterizar as diferentes famílias de Laniatores.

A grande maioria dos Laniatores basais aqui analisados apresenta ozóporo simples (ovóide), uma característica simplesiomórfica nesses grupos. A exceção foram os representantes analisados de Cranaidae e Manaosbiidae, que mostraram uma semelhança morfológica com representantes das subfamílias derivadas de Gonyleptidae, o que pode representar convergências ou sinapomorfias, e constitui um promissor e importante estudo futuro. 


\section{Resumo}

Uma peculiaridade bem conhecida da Ordem Opiliones é seu modo de defesa através do uso de substâncias químicas, sintetizadas em um par de glândulas exócrinas, cujas aberturas se localizam na lateral do prossoma e reconhecidas como ozóporo. Na sub-Ordem dos Laniatores, objeto deste estudo, alguns padrões anatômicos foram identificados a partir de imagens obtidas por meio de microscopia eletrônica de varredura. O material biológico estudado faz parte do acervo do Museu de Zoologia da Universidade de São Paulo e do Museu Nacional do Rio de Janeiro. Ozóporos posicionados na margem lateral do escudo dorsal foram encontrados nas famílias Triaenonychidae, Agoristenidae, Biantidae, Cosmetidae, Epedanidae, Escadabiidae, Fissiphalidae, Minuidae, Phalangodidae, Samoidae, Stygnidae, Stygnommatidae, Zalmoxidae, e em Podoctidae, sendo neste último quase ventral. Apenas em Cranaidae e em Manaosbiidae foram encontrados ozóporos posicionados na porção mais dorsal do prossoma. Muitos são os que apresentam apófise na coxa que de fato representam uma barreira ou apenas têm o potencial de obstrução da saída da secreção de defesa, dependendo da mobilidade da perna. Em Triaenonychidae, observou-se uma grande apófise obliterando o ozóporo em Larifugela, sendo que em Biacumontia nota-se uma apófise similar, mas de tamanho consideravelmente reduzido. Cosmetidae também apresenta uma apófise na coxa II, próxima ao ozóporo que se apresenta circular e posicionado sobre um domo. Escadabiidae, Epedanidae e Fissiphalidae apresentam a abertura do ozóporo sobre um discreto domo e canais laterais bem delimitados por uma série de tubérculos, sendo o canal vertical desta última família melhor definido. Na espécie estudada de Phalangodidae, vale ressaltar a presença 
de um canal transversal ligando, pela região dorsal, os canais laterais. Minuidae e Zalmoxidae apresentam uma abertura discreta voltada à porção posterior do corpo. 


\section{Abstract}

One of the most conspicuous characteristics of the individuals of the order Opiliones is their chemical defensive mechanisms. The chemicals are produced by a pair of exocrine glands, which open at the lateral anterior area of the body above the coxae of legs $2-3$ in Laniatores. These glands openings at the carapace are named ozopore. Previous studies of different species of the laniatorean family Gonyleptidae have shown that there are different morphologies of the ozopore area, which are directly related to different types of defensive behavior performed by the animals. This led us to study representatives of different families (the most as possible) in order to obtain an evolution overview of the ozopore area morphology within Laniatores. We used scanning electronic microscopy techniques. The ozopore is located in a dorsal position only in Manaosbiidae and Cranaidae; and at the lateral margin of the scutum in Triaenonychidae, Agoristenidae, Biantidae, Cosmetidae, Epedanidae, Escadabiidae, Fissiphalidae, Minuidae, Phalangodidae, Samoidae, Stygnidae, Stygnommatidae, Zalmoxidae and Podoctidae (in this family, it is nearly ventral). Commonly, there is a large apophysis of coxa 2 projecting above the ozopore and probably with the function of helping to define the direction of the flow of the defensive chemicals. In Triaenonychidae, a huge apophysis was observed in Larifugella (rendering difficult to observe the exact location of the ozopore), whereas in Biacumontia a remarkably smaller one was noticed. The ozopore is round in Cosmetidae, Agoristenidae (Agoristeninae) and Stygnidae (some Stygninae), it is ovoid in Biantidae (Biantinae), Epedanidae, Minuidae, Phalangodidae, Podoctidae, Stygnidae (some Stygninae), Stygnommatidae and Zalmoxidae; and a slit in the others. Regularly it is located on an integumentary dome. From each 
ozopore there is a lateral channel (or lateral row of tubercles) running posteriad. In Phalangodidae, the lateral channels present some transversal channels, which probably enhance the spreading of the defensive liquid. 


\section{Referências Bibliográficas}

ACOSTA, L. E., T. I. PORETTI \& P. E. MASCARELLI. 1993. The defensive secretions of Pachyloidellus goliath (Opiliones, Laniatores, Gonleptidae).

Bonner Zoologische Beitrage, Bonn, 44 (1-2): 19-31.

ANESHANSLEY D. J. \& T. EISNER. 1969. Biochemistry at 100ㄷ: Explosive Secretory Discharge of Bombardier Beetles (Brachinus). Department of Biochemistry, New York Medical College, New York.

BISHOP, S. C. 1950. The life of a harvestman. Nature Mag.,43:264-267, 276.

BLUM, M. S. 1981. Chemical defenses of Arthropods. Academic Press, New York.

BLUM, M. S., F. PANDOVANI \& E. AMANTE. 1968. Alkanones and terpenes in the mandibular glands of Atta species (Hymenoptera: Formicidae). Comp. Biochem. Physiol., 26: 291-298.

BLUM, M. S. \& A. L. EDGAR. 1971. 4-methyl-3-heptanone: Identification and role in opilionid exocrine secretions. Insect Biochemistry, Oxford, 1: 181-188.

BRISTOWE, W. S. 1925. Notes on the Habits of Insects and Spiders in Brazil. Transactions of the Royal Entomological Society of London, London, 1924 (34): 475-504.

CAPOCASALE, R. \& L. BRUNO-TREZZA. 1964. Biologia de Acanthopachylus aculeatus (Kirby, 1819) (Opiliones, Pachylinae). Revista de la Sociedad Uruguaya de Entomologia, Montevideo, 6: 19-32.

CLAWSON, Robert C. 1988. Morphology of Defense Glands of the Opilionids (Daddy Longlegs) Leiobunum vittatum and L. flavum (Arachnida: Opiliones: Palpatores: Phalangiidae). Journal of Morphology, 196: 363-381.

CLOUDSLEY-THOMPSON, J. L. 1958. Spider, Scorpions, Centripedes and Mites. Pergamon Press, London.

COKENDOLPHER, J. C. 1987. Observations on the defensive behaviors of a Neotropical Gonyleptidae (Arachnida, Opiliones). Revue Arachnologique, Avignon, 7 (1): 59-63.

DUfFIELD, R. M., O. OluBAJO, J. W. WHEELER \& W. A. SHEAR. 1981. Alkylphenols in the defensive secretion of the Neartic opilionid, Stygnomma spinifera (Arachnida: Opiliones). Journal of Chemical Ecology, New York, 7(2): 445-452. 
EDGAR, A. L. 1971. Studies on the biology and ecology of Michigan Phalangida (Opiliones). Miscellaneous Publications Museum of Zoology, University of Michigan, Ann Arbor, 144: 1-64.

EDMUNDS, M. 1974. Defense in Animals: A Survey of Antipredator Defenses. Longman, Harlow.

EISNER, T. 1970. In "Chemical Ecology" (E. Sondheimer and J. B. Simeone, eds.) Academic Press, New York, p. 157-217.

EISNER, T. \& J. MEINWALD. 1966. Defensive Secretions of Arthropods. Science. 153(3742): 1341-1350.

EISNER, T., F. KLUGE, J. E. CARREL \& J. MEINWALD. 1971. Defense of Phalangid: liquid repellent administred by leg dabbing. Science, Washington, 173 (3997): 650-652.

EISNER, T., T. H.JONES, K. HICKS, R. E. SILBERGLIED \& J. MEINWALD. 1977. Quinones and phenols in the defensive secretions of neotropical opilionids. Journal of Chemical Ecology, New York, 3 (3): 321-329.

EISNER, T., D. ALSOP \& J. MEINWAD. 1978. Secretions of Opilionids, Whip Scorpions and Pseudoscorpions. Pp. 87-99 in Handbook of Experimental Pharmachology (Arthropod Venoms), Vol. 48 (S. Bettini, ed.). Springer-Verlag, Berlin.

EISNER, T., C. ROSSINI, A. GONZÁLEZ \& M. EISNER. 2004. Chemical defense of an opilionid (Acanthopachylus aculeatus). The Journal of Experimental Biology, 207: 1313-1321.

EKPA, O., J. W. WHEELER, J. C. COKENDOLPHER \& R. M. DUFFIELD. 1984. $\mathrm{N}, \mathrm{N}$-dimethyl- $\beta$-phenylethylamine and bornyl esters from the harvestman Sclerobunus robustus (Arachnida: Opiliones). Tetrahedron Letters, Oxford, 25 (13): 1315- 1318.

EKPA, O., J. W. WHEELER, J. C. COKENDOLPHER \& R. M. DUFFIELD. 1985. Ketones and Alcohool in the defensive secretion of Leiobunum townsendi weed and a review of the known exocrine secretions of Palpatores (Arachnida: Opiliones). Comp. Biochem. Physiol., 81B(3): 555-557.

ESTABle, C., M. I. ARDAO, N. P. BRASIL, \& L. F. FIESER. 1955. Gonyleptidine. Journal of the American Chemical Society, Washington, 77: 4942.

FIESER, L. F. \& M. I. ARDAO. 1956. Investigation of the chemical structure of the Gonyleptidine. Journal of the American Chemical Society, Washington, 78 (4): 774-781. 
GIRIBET, G., G. D. EDGECOMBE, W. C. WHEELER \& C. BABBIT. 2002. Phylogeny and systematic position of Opiliones: a combined analysis of chelicerate relationships using morphological and molecular data. Cladistics, 18: 5-70.

GIRIBET, G. \& A. B. KURY. 2007. Chapter 3. Phylogeny and Biogeography. p. 6297. In: PINTO-DA-ROCHA, R., G. MACHADO \& G. GIRIBET (Organizadores), Harvestmen. The Biology of Opiliones. Harvard University Press. 597 pp.

GNASPINI, P. \& A. J. CAVALHEIRO. 1998. Chemical and behavioral defenses of a neotropical cavernicolous harvestman: Goniosoma spelaeum (Opiliones, Laniatores, Gonyleptidae). The Journal of Arachnology, New York, 26: 81-90.

GNASPINI, P. \& M. R. HARA. 2007. Chapter 10. Defense Mechanisms. p. 374-399. In: PINTO-DA-ROCHA, R., G. MACHADO \& G. GIRIBET (Organizadores), Harvestmen. The Biology of Opiliones. Harvard University Press. 597 pp.

HARA, M. R. \& P. GNASPINI. 2003. Comparative study of the defensive behavior and morphology of the gland opening area among harvestmen (Arachnida, Opiliones, Gonyleptidae) under a phylogenetic perspective. Arthropod Structure \& Development, 32: 257-275.

HARA, M. R., A. J. CAVALHEIRO, P. GNASPINI \& SANTOS D. Y. A. C. 2005. A comparative analysis of the chemical nature of defensive secretions of Gonyleptidae (Arachnida: Opiliones: Laniatores). Biochemical Systematics and Ecology, 33: 1210-1225.

HOLMBERG. R. G. 1970. The odoriferus glands of some Palpatores Phalangida (Opiliones) (Arachnida). M.Sc. Thesis, University Of Saskatchewan, Saskatoon, Canada.

HOLMBERG. R. G. 1986. The scent glands of Opiliones: a review of their function. Pp. 131-133 in Proceedings of the 9th International Arachnological Congress (W.G. Eberhard, Y.D. Lubin, \& B. C. Robinson, eds.). Smithsonian Institution Press, Washington, DC.

JONES, T. H., W. E. CONNER, A. F. KLUGE, T. EISNER \& J. MEINWALD. 1976. Defensive substances of Opilionids. Experientia, Basel, 32 (10): 1234-1235.

JONES, T. H., J. MEINWALD, K. HICKS \& T. EISNER. 1977. Characterization and synthesis of volatile compounds from the defensive secretions of some "daddy longlegs" (Arachnida: Opiliones: Leiobunum spp.). Proceedings of the National Academy of Sciences of the United States of America, Washington, 74 (2): 419-422.

JUBERTHIE, C. 1961a. Données sur la biologie des Ischyropsalis C.L.K. (Opilions, Palpatores, Ischyropsalidae). Ann. Spéléol., 16:381-395. 
JUBERTHIE, C. 1961b. Étude des Opilons Cyphophthalmes (Arachnides) de Portugal: descriptions d' Odontosiro lusitanictus gen.n., sp.n. Bull. Mus. Hist. Nat. Paris, 33:512-519.

KREBS, J. R. \& N. B. DAVIES. 1966. An Introduction to Behaviour Ecology. Blackwell, Oxford.

LAWRENCE, R. F. 1938. The odoriferous glands of some South African Harvestspiders. Transactions of the Royal Society of South Africa, 25 (4): 333-342.

MACHADO, G. 2002. Maternal care, defensive behavior, and sociality in neotropical Goniosoma harvestmen (Arachnida, Opiliones). Insects Sociaux, 49: 388-393.

MACHADO, G. \& C. H. F. VASCONCELOS. 1998. Multi-Species aggregations in neotropical harvestmen (Opiliones, Gonyleptidae). Journal of Arachnology, 26: 389-391.

MACHADO, G. \& P. S. OLIVEIRA. 1998. Reproductive biology of the neotropical harvestman (Goniosoma longipes) (Arachnida, Opiliones: Gonyleptidae): mating and oviposition behaviour, brood mortality, and parental care. Journal of Zoology, 246: 359-367.

MACHADO, G., R. L. G. RAIMUNDO \& P. S. OLIVEIRA. 2000. Daily activity schedule, gregariousness, and defensive behaviour in the Neotropical harvestman Goniosoma longipes (Opiliones, Gonyleptidae). Journal of Natural History, 34: 587-596.

MACHADO, G., V. BONATO \& P. S. OLIVEIRA. 2002. Alarm communication: a new function for the scent-gland secretion in Harvestmen (Arachnida: Opiliones). Naturwissenschaften, 89: 357-360.

MACHADO, G., P. C. CARRERA, A. POMINI \& A. J. MARSAIOLI. 2005. Chemical Defense in Harvestmen (Arachnida, Opiliones): do benzoquinone secretions deter invertebrate and vertebrate predators? Journal of Chemical Ecology, New York, 31 (11): 2519-2539.

MACHADO, G., R. PINTO-DA-ROCHA \& G. GIRIBET. 2007. Chapter 1. What are harvestmen? p. 1-13. In: PINTO-DA-ROCHA, R., G. MACHADO \& G. GIRIBET (Organizadores), Harvestmen. The Biology of Opiliones. Harvard University Press. 597 pp.

MAURY, E. A. 1987. Triaenonychidae sudamericanos. IV. El género Triaenonychoides H. Soares 1968 (Opiliones, Laniatores). Boletim de la Sociedad de Biologia de Concepción, 58: 95-106.

MEINWALD, J., A. F. KLUGE, J. E. CARREL \& T. EISNER. 1971. Acyclic ketones in the defensive secretion of a "daddy longlegs" (Leiobunum vittatum) 
(Arachnida/Opiliones). Proceedings of the National Academy of Sciences of the United States of America, Washington, 68 (7): 1467-1468.

MORA, G. 1990. Paternal care in a neotropical harvestman, Zygopachylus albomarginis (Arachnida, Opiliones: Gonyleptidae). Animal Behavior, 39: 582593.

MURPHREE, C. S. 1988. Morphology of the dorsal integument of ten opilionid species (Arachnida, Opiliones). The Journal of Arachnology, New York, 16: 237-252.

PINTO-DA-ROCHA, R. 2002. Systematic review and cladistic analysis of the Caelopyginae (Opiliones: Gonyleptidae). Arquivos de Zoologia, São Paulo, 36: 357-464.

PINTO-DA-ROCHA, R. \& G. GIRIBET. 2007. Chapter 4. Taxonomy. p. 88-246. In: PINTO-DA-ROCHA, R., G. MACHADO \& G. GIRIBET (Organizadores), Harvestmen. The Biology of Opiliones. Harvard University Press. 597 pp.

RAMIRES, E. N., \& A. A. GIARETTA. 1994. Maternal care in a neotropical harvestman, Acutisoma proximum (Opiliones, Gonyleptidae). J. Arachnol., 22: 179-180.

ROACH, B., T. EISNER \& J. MEINWALD. 1980. Defensive substances of Opilionids. Journal of Chemical Ecology, New York, 6 (2): 511-516.

RODRIGUEZ, C. A. \& S. GUERRERO. 1976. La Historia Natural y el Comportamiento de Zygopachylus albomarginis (Chamberlain) (Arachnida, Opiliones: Gonyleptidae). Biotropica, 8 (4): 242-247.

SHEAR, W. A. 1982. Opiliones. Pp. 104-110 in Sypnopsis and Classification of Living Organisms, vol. 2 (S.P. Parker, ed.). McGraw-Hill, New York.

SHULTZ, J. W. 1990. Evolutionary morphology and phylogeny of Arachnida. Cladistics, 6:1-38.

WHEATHERSTON, J. \& J. E. PERCY. 1970. Arthropod defensive secretions in Chemicals Controlling Insect Behaviour. Ed. Beroza, M. pp. 95-144.

WIEMER, D. F., K. HICKS, J. MEINWALD \& T. EISNER. 1978. Naphthoquinones in defensive secretion of an opilionid. Experientia, Basel, 34 (8): 969-970.

WILLEMART, R. H. \& P. GNASPINI. 2004. Breeding biology of the cavernicolous harvestman Goniosoma albiscriptum (Arachnida, Opiliones, Laniatores): sites of oviposition, egg batches characterisitics and subsocial behaviour. Invertebr. Reprod. Dev. 45: 15-28. 
WILLEMART, R. H. 2002. Cases of intra- and inter-specific food competition among Brazilian harvestmen, in captivity (Opiliones, Laniatores, Gonyleptidae). Revue Arachnologique, 14 (4): 49-58. 